\title{
Alain Joannes, Le journalisme à l'ère électronique
}

Paris, Vuibert, coll. Lire Agir, 2007, 247 p.

Pierre Morelli

\section{OpenEdition}

\section{Journals}

Édition électronique

URL : http://journals.openedition.org/questionsdecommunication/1564

DOI : 10.4000/questionsdecommunication. 1564

ISSN : 2259-8901

\section{Éditeur}

Presses universitaires de Lorraine

Édition imprimée

Date de publication : 1 décembre 2008

Pagination : 355-357

ISBN : 978-2-86480-981-4

ISSN : 1633-5961

\section{Référence électronique}

Pierre Morelli, «Alain Joannes, Le journalisme à l'ère électronique », Questions de communication [En

ligne], 14 | 2008, mis en ligne le 23 janvier 2012, consulté le 22 septembre 2020. URL : http:// journals.openedition.org/questionsdecommunication/1564; DOI : https://doi.org/10.4000/ questionsdecommunication. 1564

Ce document a été généré automatiquement le 22 septembre 2020

Tous droits réservés 


\title{
Alain Joannes, Le journalisme à l'ère électronique
}

Paris, Vuibert, coll. Lire Agir, 2007, 247 p.

\author{
Pierre Morelli
}

\section{RÉFÉRENCE}

Alain Joannes, Le journalisme à l'ère électronique, Paris, Vuibert, coll. Lire Agir, 2007, $247 \mathrm{p}$.

1 Depuis l'invention de la presse de masse au XIX ${ }^{\mathrm{e}}$ siècle, le journalisme a connu de nombreuses crises qu'il a surmontées tant bien que mal. Toutefois, la dernière crise en date est cruciale dès lors qu'elle affecte de manière profonde et durable toutes les phases et tous les acteurs impliqués - destinataires compris - dans la construction et à la diffusion de l'information. En 247 pages abondamment illustrées par des cas précis empruntés à l'actualité récente - ou particulièrement exemplaire - et complétées par de nombreux tableaux explicatifs, des fiches techniques et pratiques, le journaliste Alain Joannes mobilise une expérience professionnelle fort riche et offre un guide destiné aux professionnels de l'information et aux étudiants en journalisme, désireux de suivre au plus près l'évolution de la presse et de ses métiers. Contrairement à ce que le début du titre laisserait à penser, la réflexion ne se limite ni au journalisme électronique sur le Web, ni à une nouvelle forme professionnelle héritée du développement actuel de l'informatique en réseau. À travers dix chapitres découpés en sous-chapitres, l'auteur propose une présentation claire et panoptique des situations professionnelles à venir, un exposé étayé par de nombreuses références scientifiques que le recours incessant à des "zooms » explicatifs - définitions claires et succinctes précédées d'un logo représentant une loupe - et à des exemples synthétiques - faits d'actualité qui illustrent la situation - rend pédagogique.

2 Alain Joannès postule que l'avènement des technologies de l'information et de la communication en réseau modifie irrémédiablement les conditions dans lesquelles 
s'exerceront les «savoir-faire fondamentaux du journalisme -collecter, vérifier, analyser, structurer, diffuser » (p. 9). En matière de traitement de l'information, ceux-ci sont à repenser à l'aune du développement technologique. Les journalistes gagneront à s'approprier les modalités narratives nouvelles, plus en phase la multiplication des supports et des formes d'accès à l'information, avec la fragmentation du temps, la segmentation des cibles et la volatilité qui caractérise le comportement de l'individu hypermoderne. Si la profusion de sources, l'ubiquité, la diversification des modes de diffusion, et plus particulièrement l'instantanéité dans la transmission de l'information peuvent être considérées comme des contraintes, elles représentent néanmoins des atouts qu'il conviendra désormais de maîtriser. Une évolution des mentalités et des pratiques professionnelles s'annonce donc.

3 Les trois défis à relever que l'auteur présente dans le premier chapitre (pp.11-27) surmonter le risque d'« engourdissement corporatiste", affronter les situations nouvelles générées par l'inéluctable "concurrence des supports", intéresser "des audiences fuyantes» - distribuent les enjeux tout au long du schéma communicationnel. En amont, sont concernées la frilosité d'une majorité de journalistes peu enclins à changer de média, puis la mécompréhension de l'intérêt de l'internet, l'introduction $d u$ "concept d'information continue» à l'aube du développement de la toile mondiale. En aval, on constate l'apparition puis la généralisation d'une forme d'individualisme contemporain que l'auteur qualifie d'«hypermoderne" causée par un contexte de réception détérioré par le manque d'appétence informationnelle - "l'inforexie des Français» (p. 23) et stimulée par la segmentation des emplois du temps rongés les contraintes imposées par les nouveaux modes de vie coupables, selon Alain Joannès, de l'instauration d'une "société "chronophage" » (p. 24) au sein de laquelle le journaliste est désormais tenu de "se donner les moyens de s'adresser efficacement à cette nouvelle sensibilité narcissique obsédée par la performance et l'excès, hantée par l'incertitude et la perte d'identité " (p. 27). Pour l'auteur, l'avenir de la profession passe par une indispensable reconfiguration du métier (pp. 29- 48). La mutation qui s'impose repose en grande partie sur la remise en question de la nature de l'information telle qu'elle est délivrée depuis le siècle dernier. En effet, représenter de manière linéaire les événements c'est « se condamner à appauvrir l'information en essayant de l'ajuster à la fragmentation du temps et au comportement volage de l'individu hypermoderne» (p. 30). A contrario, acquérir le réflexe rich media, c'est-à- dire mobiliser tout moyen d'expression pour décrire, raconter ou expliquer un événement «transforme le défi de la complexité en un atout puissant: plus un événement est riche, plus il justifie une forme attractive " (p. 34). Autre avantage, l'adoption de techniques narratives dynamise le rapport à l'information, laquelle sera traitée tantôt de manière sonore, tantôt visuelle ou textuelle en fonction de sa spécificité et en fonction des contraintes de mise en ligne. Acquérir le réflexe rich media signifie accepter une modification de la culture professionnelle des journalistes. Il ne s'agit non pas d'une injonction à couvrir ou à transformer l'information de manière systématiquement multimodale, mais au contraire à anticiper une nouvelle dimension dans la mise en forme et la réception de l'information. Il est alors question d'installer une dimension temporelle qui facilite l'assimilation et ouvre une profondeur documentaire que les médias traditionnels ne facilitaient pas: "De même qu'un journaliste n'est pas tenu de savoir déployer une information dans tous les modes d'expression, rien n'oblige une rédaction à tout transformer tout de suite en contenus rich media. Le plus important est de raisonner 
d'emblée dans la perspective rich media et d'anticiper les enrichissements possibles " (p. 37). L'adoption de ce réflexe ne constitue pourtant pas la seule réponse car, plus que jamais, il est indispensable d'« envisager une autre manière de traiter l'information pour la rendre plus riche et plus attractive [ce qui] suppose que le journalisme accepte la notion de productivité [...] a priori répulsive car elle est souvent génératrice de pénibilité, de stress et de chômage » (p. 48).

On l'aura compris, les modifications affectent tous les niveaux de la pratique professionnelle. Le journaliste est tenu de « réinventer la collecte " par l'usage raisonné des outils contemporains et à travers des dispositifs qui incluent le hasard (voir le chapitre trois). En outre, les pièges de l'internet l'obligent à vérifier l'information au moyen de nouvelles méthodes et d'outils adaptés, au risque de participer de la prolifération de contenus informationnels "piégés » dont une typologie est présentée dans le chapitre quatre (rumeurs, légendes urbaines, hoaxes, fakes, buzz...), l'analyse des documents et la construction d'une documentation étant développées dans les chapitres cinq et six.

5 Mais les modifications concernent aussi le mode de fonctionnement des rédactions. Plus précisément, l'enjeu consiste, selon Alain Joannès, à créer puis développer les conditions optimales pour que s'exerce la nécessaire polyvalence des journalistes du futur. Dans le chapitre sept («Organiser une rédaction»), l'auteur postule que les rédactions doivent, à cet effet, pouvoir « se reconfigurer à tout moment » (p. 143), et le journalisme gagnerait à réaliser « la symbiose de l'individualisme, gage d'originalité et de diversité, et du travail coopératif, source d'efficacité et de crédibilité » (ibid.). Ainsi l'auteur esquisse-t-il des solutions à même de rendre l'organisation des rédactions plus performante et d'optimiser leur fonctionnement. Soucieux de porter sa réflexion sur les différentes phases de la chaîne communicationnelle, il renvoie la question de la diffusion au chapitre suivant. Les différentes formes que prend l'information (et qui relèvent de la question des genres journalistiques) sont alors passées en revue, ce à l'aune de la spécificité des médias numériques et des habitudes avérées de lecture. Plus qu'une réflexion formelle, l'auteur s'emploie à resituer toutes les situations dans une perspective communicationnelle. Par exemple, le lecteur apprendra que "les alertes » permettent d'entretenir une "relation de confiance entre la rédaction et ses audiences » (p. 168), que les brèves complètent les alertes et « annoncent l'existence d'articles factuels» (p. 171). Quant aux articles, ils sont au cœur de l'information (p. 172) tandis que les reportages ont pour principale vertu de crédibiliser la rédaction. Dès lors, l'interview et le commentaire doivent respectivement être déclinés en rich media, conformément à « la réceptivité supposée des médianautes» (p. 175)... La stratégie de diffusion doit donc être «multicanal » (p. 183) et appelle l'intégration des potentialités offertes par l'interactivité au service d'un dialogue nourri avec le lectorat car, comme l'explique Alain Joannès dans l'avant-dernier chapitre, l'interactivité permet de comprendre mieux et en permanence les audiences et qu'avec la montée en puissance de l'internet, à partir de 1997, s'est installée l'injonction permanente du temps réel véritable défi à relever. Dans ce contexte, "chercher les bonnes réponses à ce défi oblige à s'interroger sur ce qu'il convient de diffuser et à quel moment» (p. 185). C'est précisément le sens de la réflexion traitant de la place, du rôle et des effets de l'interactivité qui, en retour, délivre des éléments de compréhension fine des audiences (pp.195-202), au risque de produire plus de commentaires que d'articles et de reproduire, chez les « "journalistes citoyens" tous les comportements qu'il condamnent chez les journalistes professionnels» (p. 202). Ce qui se joue n'est rien moins que la 
construction de «connivences équilibrées. [Dès lors, le] journaliste est en mesure, non seulement d'écouter et de mieux comprendre ceux qui ne demandent qu'à lui faire confiance, mais aussi de travailler à restaurer la fiabilité de la profession au sein de communautés de connaissance qui réfléchissent sur l'actualité» (ibid.). Ainsi de nouveaux défis s'annoncent-ils. Ils sont listés dans un dernier chapitre déclinant la "prolifération de l'offre de contenus» (p. 204), l'accentuation de la mobilité des destinataires, la transformation qui confère aux réseaux des qualités d'intelligences susceptibles de toucher des audiences toujours plus "pressurées et fragmentées» (p. 213).

Enfin, le souci d'offrir une vision claire et panoptique des mutations qui saisissent le journalisme bénéficie de la mise en place d'un blog (http://www.journalistiques.fr/) dans lequel Alain Joannès développe, prolonge et ouvre sa réflexion aux lecteursinternautes. Cette initiative est à saluer. La lecture de ce guide captivera donc aussi bien les professionnels que les néophytes. Les premiers trouveront des pistes et des informations rapidement exploitables, les seconds auront accès à une vision détaillée et éclairée de la réalité professionnelle.

\section{AUTEURS}

\section{PIERRE MORELLI}

CREM, Université Paul Verlaine-Metz, morelli@univ-metz.fr 